\section{A test of independence of dimensions in multidimensional scaling}

\author{
KARL WENDER \\ Technische Hochschule, Darmstadt, Germany
}

Models for multidimensional scaling use metric spaces with additive difference metrics. Two important properties of additive difference metrics are decomposability and intradimensional subtractivity. A prediction was derived from these properties and tested experimentally. Rectangles varying in area and shape served as stimuli. Dissimilarity judgments were obtained by both rating and pair comparison procedures. The assumptions of the model were violated by most of the Ss. Apparently this violation was due to an interaction between the two dimensions.

In recent years models for multidimensional scaling have been advanced in several ways. One of the major contributions is that of Beals and Krantz (1967), Beals, Krantz, and Tversky (1968), and Tversky and Krantz (1969), who introduced a system of axioms for ordinal multidimensional scaling and proved appropriate representation and uniqueness theorems. In particular, these axioms show how restrictive are the assumptions on which commonly used multidimensional scaling procedures are based. Not all of the axioms are testable in the sense of Pfanzagl $(1968$, p. 108), but at least some of them allow one to design an experiment which eventually may show that the axioms $d c$ not hold under particular circumstances.

So far there are few empirica investigations of the axioms. The onls published study known to the presen author is that of Tversky and Krantz (1969), who tested predictions derivec from the axioms for interdimensiona: additivity. Using schematic faces as stimuli Tversky and Krantz inferred from their data that interdimensional additivity might well be satisfied but, as they noted, this conclusion is valid only for their type of stimuli and configuration. In fact, the study involved only very few stimuli presented in a special, regular configuration. This configuration may have biased their results toward not violating the assumptions.

The present study investigated a similar property of the model, using different stimuli and a different configuration. Furthermore, the hypothesis tested was that with the same kind of stimuli but with different configurations the model might be violated in different ways due to context effects.

Metric spaces commonly in use with multidimensional scaling procedures are characterized by additive segments and additive difference metrics. This paper is concerned with a special property of additive difference metrics.
Beals, Krantz, and Tversky (1968) write the general model of additive difference metrics in the following way

$$
\mathrm{d}(\mathrm{x}, \mathrm{y})=\mathrm{F} \sum_{\mathrm{i}}^{\mathrm{n}} \Phi_{\mathrm{i}}\left(\left|\mathrm{X}_{\mathrm{i}}-\mathrm{Y}_{\mathrm{i}}\right|\right)
$$

where $d(x, y)$ is the subjective difference between two stimuli, $x$ and $y . X_{i}=f_{i}\left(x_{i}\right)$ and $Y_{i}=f_{i}\left(y_{i}\right)$ are the coordinates of the corresponding points in subjective space. The $x_{i}$ and $y_{i}$ are their values on the "relevant" physical dimensions. The $f_{i}$ are real valued functions, sometimes called psychophysical funtions. The essence of the models lies in three properties: (1) decomposability; (2) interdimensional additivity; and (3) intradimensional subtractivity. Decomposability requires that there be no interaction between the dimensions of the subjective space, i.e., the dimensions contribute independently to the overall distance. In Eq. 1 this is expressed by the fact that the arguments of the $\Phi$ functions for each $i$ belong to the same ith dimension. Intradimensional subtractivity specifies that on each dimension the absolute value of the difference between the corresponding coordinates is computed. To satisfy interdimensional additivity these contributions are combined by addition after some monotone transformation, $\Phi_{i}$. A further monotone transformation, $F$, gives the distance between the points.

The present paper is primarily concerned with decomposability and subtractivity. The general model for these properties may be written as

$$
\begin{aligned}
d(x, y)= & F\left[\Phi_{1}\left(\left|X_{1}-Y_{1}\right|\right),\right. \\
& \left.\cdots, \Phi_{n}\left(\left|X_{n}-Y_{n}\right|\right)\right]
\end{aligned}
$$

where all symbols are defined as before, except $F$, which is now a function of $n$ variables.
From Eq. 2 it follows immediately: If $x$, $y, u, v$ are stimuli, with $x_{i}=y_{i}$ and $u_{i}=v_{i}$ for some $i$, and with $x_{j}=u_{j}$ and $y_{j}=v_{j}$ for all $\mathrm{j} \neq i$, then: $d(\mathrm{x}, \mathrm{y})=\mathrm{d}(\mathrm{u}, \mathrm{v})$. This property of the model is the one tested in the following experiment.

In an unpublished experiment, the present author found that a multidimensional scaling study using the complete method of triads (Torgerson, 1958) with rectangles varying in shape and area resulted in an apparent interaction between these two dimensions. That is, subjective dissimilarities which should be due only to differences in area seemed to depend also on differences in shape. This is a violation of decomposability. Furthermore, the data suggested that dissimilarity judgments in some region of the space might be influenced by the degree to which this region is represented in the set of stimuli used throughout the experiment. If one region in the space is represented by relatively many stimuli, Ss may respond to them by making larger dissimilarity judgments as compared with judgments about stimuli from less well represented regions. Somewhat similar results have been found in unidimensional scaling, e.g., Stevens, 1959. Since the study mentioned above was conducted for a different purpose, the present experiment was designed to investigate this hypothesis more accurately.

\section{METHOD}

The stimuli used were rectangles varying in area and shape. Shape was defined as the ratio of height to width. As shown in Fig. 1, three sets of stimuli were prepared.

Stimuli 1-10 were common to all three sets, $a, b$, and $c$. Sets $b$ and $c$ contained additional stimuli, which were more like squares in Set $b$ and more elongated in Set $c$.

The rectangles were cut from heavy white paper, photographed, and shown by a slide projector. They appeared as dark figures on a bright background.

Two types of dissimilarity judgments were obtained: rating scale judgments and pair comparisons. In the rating scale experiment, two stimuli at a time were shown, and Ss were asked to judge the overall difference on a rating scale running from 0 for "no difference" to 8 for "very large difference." The following pairs of stimuli were prepared for display. Set a: $(1,6),(2,7),(3,8),(4,9),(5,10)$; Set $b$ : same as for Set a plus $(11,23),(15,19)$, $(16,20),(12,24) ;$ Set $c$ : same as for Set a plus $(13,25),(17,21),(18,22),(14,26)$.

The two rectangles of each pair appeared in the slides in upright position, with their midpoints at the same height and separated by a distance which was about three times 

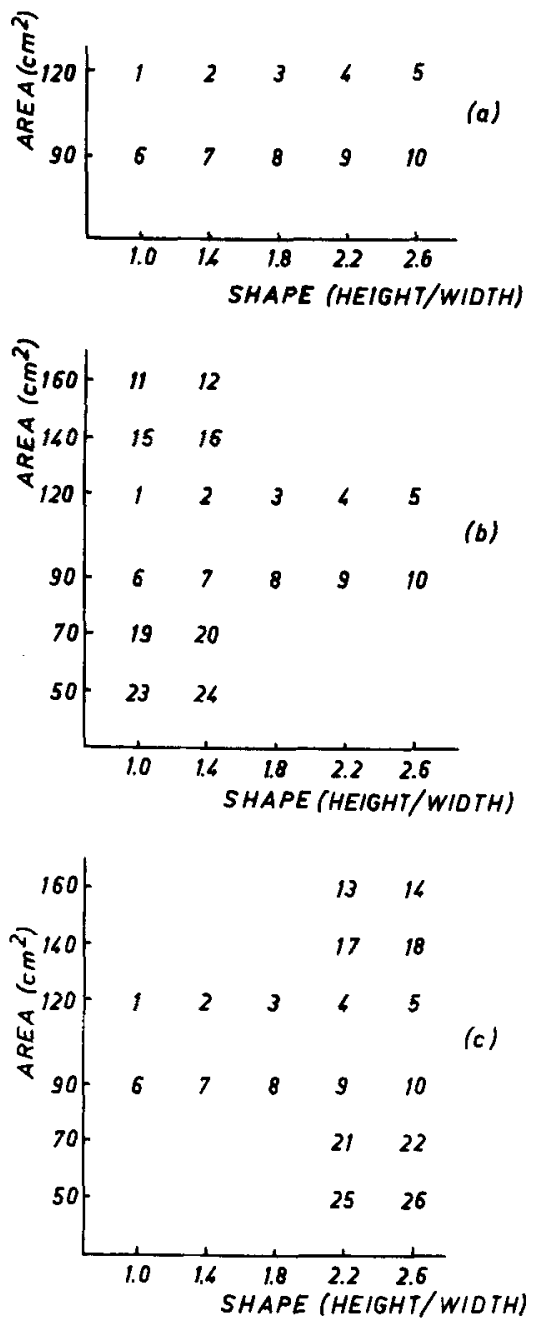

Fig. 1. Stimulus configurations used in Sets $a, b$, and $c$.

their width. Each stimulus pair was photographed six times: three times each in reversed left-right position. This gave 30 slides for Experiment a and 54 slides for Experiments $b$ and $c$. The slides were presented in random sequence.

Each of the experiments began with 30 training trials, using the first $\mathbf{3 0}$ slides. The data for these training trials were excluded from the analysis. During the experiments proper each slide was judged twice by all Ss, resulting in 12 judgments on each stimulus pair by each $S$.

For the pair comparison experiment, the same stimulus pairs were used as above, but not all possible pairs of pairs were presented. The slides showed two stimuli: one pair on the left side and one on the right side separated by a dark line. The Ss had to judge which of the two pairs showed the larger overall difference.

For Experiment d, all 10 possible combinations of pairs from Set a were photographed six times, left-right position

Table 1

Mean Ratings of Difference and $\chi^{2}$ Values

\begin{tabular}{|c|c|c|c|c|c|c|c|}
\hline \multirow[b]{2}{*}{ Subject } & \multicolumn{5}{|c|}{ Stimulus Pair } & \multirow{2}{*}{\multicolumn{2}{|c|}{$x^{2}$}} \\
\hline & $(1,6)$ & $(2,7)$ & $(3,8)$ & $(4,9)$ & $(5,10)$ & & \\
\hline \multicolumn{8}{|c|}{ Set A } \\
\hline 1 & 3.4 & 3.6 & 5.3 & 6.0 & 7.0 & 23.9 & $x$ \\
\hline 2 & 5.3 & 5.1 & 4.7 & 5.9 & 6.6 & 7.5 & \\
\hline 3 & 4.3 & 5.5 & 5.0 & 5.3 & 6.2 & 3.8 & \\
\hline 4 & 4.0 & 4.2 & 5.7 & 6.4 & 6.2 & 31.0 & $x$ \\
\hline 5 & 5.1 & 4.8 & 5.3 & 4.8 & 4.4 & 3.7 & \\
\hline \multicolumn{8}{|c|}{ missing data } \\
\hline 7 & 2.3 & 3.1 & 5.9 & 6.6 & 6.3 & 28.8 & $x$ \\
\hline 8 & 3.3 & 4.1 & 4.5 & 5.3 & 5.5 & 29.2 & $x$ \\
\hline 9 & 1.5 & 3.7 & 4.4 & 7.3 & 7.7 & 44.5 & $\mathrm{x}$ \\
\hline 10 & 3.7 & 4.2 & 5.8 & 5.1 & 5.3 & 8.1 & $x$ \\
\hline 11 & 4.0 & 4.3 & 4.7 & 4.6 & 5.5 & 6.0 & \\
\hline \multicolumn{8}{|c|}{ Set B } \\
\hline 1 & 1.6 & 2.7 & 2.5 & 2.8 & 2.6 & 12.8 & \\
\hline 2 & 1.0 & 2.1 & 2.3 & 2.4 & 2.7 & 30.5 & $x$ \\
\hline 3 & 2.6 & 3.3 & 3.1 & 2.8 & 3.3 & 2.7 & \\
\hline 4 & 2.8 & 2.8 & 3.1 & 2.6 & 3.0 & 3.8 & \\
\hline 5 & 1.7 & 2.3 & 2.9 & 3.1 & 3.7 & 23.4 & $\mathrm{X}$ \\
\hline 6 & 1.2 & 2.1 & 2.0 & 2.8 & 2.8 & 27.2 & $\mathbf{x}$ \\
\hline 7 & 1.5 & 2.0 & 2.2 & 1.8 & 2.2 & 1.6 & \\
\hline 8 & 3.3 & 3.9 & 3.9 & 3.0 & 3.2 & 15.6 & $x$ \\
\hline 9 & 1.8 & 2.3 & 1.8 & 1.7 & 2.3 & 2.7 & \\
\hline 10 & 1.8 & 1.8 & 1.0 & 2.1 & 2.8 & 8.2 & \\
\hline 11 & 1.0 & 2.3 & 2.5 & 2.5 & 2.5 & 5.0 & \\
\hline \multicolumn{8}{|c|}{ Set $C$} \\
\hline 1 & 2.2 & 2.2 & 2.3 & 2.3 & 2.3 & 0.8 & \\
\hline 2 & 1.2 & 1.8 & 1.8 & 1.8 & 1.9 & 5.4 & \\
\hline 3 & 2.3 & 3.4 & 3.2 & 3.4 & 3.4 & 10.2 & \\
\hline 4 & 2.6 & 2.6 & 2.8 & 2.7 & 2.8 & 1.0 & \\
\hline 5 & 1.4 & 1.8 & 2.1 & 2.3 & 2.0 & 6.7 & \\
\hline 6 & 1.7 & 2.0 & 2.1 & 2.1 & 2.3 & 4.0 & \\
\hline 7 & 1.4 & 2.4 & 2.5 & 2.4 & 2.3 & 9.7 & \\
\hline 8 & 3.7 & 4.8 & 5.0 & 4.8 & 3.7 & 23.1 & \\
\hline 9 & 1.4 & 3.3 & 3.3 & 2.7 & 2.3 & 10.8 & \\
\hline 10 & 2.3 & 1.9 & 1.8 & 1.9 & 1.8 & 2.0 & \\
\hline 11 & 2.5 & 2.5 & 2.2 & 2.3 & 2.3 & 2.3 & \\
\hline
\end{tabular}

counterbalanced. The 60 slides were presented in random sequence and judged twice so that again 12 judgments on each stimulus combination were obtained. Sets e and $f$ were made from Sets $b$ and $c$, respectively. From the nine pairs, 36 quadruples were photographed twice, left-right position counterbalanced. This series of 72 slides was judged six times by each $\mathrm{S}$, resulting in 12 judgments on all stimulus combinations.

The Ss were 11 nonpsychology students who were paid for their participation. Each $S$ was given all sets (a-e) in individual sessions. The sessions for Sets a-d took 30 to $45 \mathrm{~min}$. Experiments $\mathrm{e}$ and $\mathrm{f}$ were divided into three parts, each of which took the same time. Each S had a total of 10 sessions on 10 different days, each session starting with 30 training trials. Slide presentation was controlled by an electronic timing device. Slides were shown by a slide projector for $8 \mathrm{sec}$, during which time the $\mathrm{Ss}$ responded by pressing a button. The judgments were punched on a paper tape.

\section{RESULTS}

The data were analyzed separately for each S. Only those stimulus pairs which were common to all six subexperiments were included in the analysis, i.e., the pairs $(1,6)$ through $(5,10)$.

Individual data for the rating scale experiment consisted of a 5 by 12 matrix with integers between 0 and 8 as entries. If Eq. 2 holds, the row means should vary only due to random fluctuations. To test Eq. 2, the Kruskal-Wallis $H$ statistic was computed, since ordinal multidimensional scaling requires only ordinal scale data. In Table 1, the resulting chi-square values for each $S$ are given together with the arithmetic means of the difference judgments for each of the stimulus pairs: All chi-square values exceeding 15.3 (indicated by an asterisk) are significant at the .01 level.

In the pair comparison experiment, each slide showed one pair of stimuli that looked more like squares, the other pair being relatively slender. If a $S$ chose the pair consisting of the thinner rectangles as having the larger overall difference, this judgment was scored " 1 ," and " 0 " otherwise. Thus the data for each $S$ consist of a 5 by 5 pair comparison matrix with the main diagonal left empty. The entries of the matrix show how often the thinner pair was chosen over the more square one. 
Table 2

Number of Times Each Stimulus Pair Was Preferred to Any Other Pair and Sums of the Pair Comparison Matrices

\begin{tabular}{|c|c|c|c|c|c|c|c|}
\hline \multirow{2}{*}{$\begin{array}{l}\text { Sub- } \\
\text { ject }\end{array}$} & \multicolumn{5}{|c|}{ Stimulus Pair } & \multirow{2}{*}{\multicolumn{2}{|c|}{ Sum }} \\
\hline & $(1,6)$ & $(2,7)$ & $(3,8)$ & $(4,9)$ & $(5,10)$ & & \\
\hline & \multicolumn{5}{|c|}{ Set D } & & \\
\hline 1 & 0 & 14 & 24 & 34 & 48 & 118 & $\mathbf{x}$ \\
\hline 2 & 34 & 28 & 20 & 19 & 19 & 43 & $\mathrm{x}$ \\
\hline 3 & 45 & 26 & 22 & 17 & 10 & 25 & $\mathrm{x}$ \\
\hline 4 & 1 & 12 & 25 & 34 & 48 & 118 & $\mathbf{x}$ \\
\hline 5 & 3 & 11 & 25 & 34 & 47 & 116 & $\mathbf{x}$ \\
\hline 6 & 47 & 24 & 19 & 17 & 13 & 29 & $\mathbf{x}$ \\
\hline 7 & 34 & 29 & 24 & 18 & 15 & 41 & $\mathrm{x}$ \\
\hline 8 & 0 & 12 & 24 & 36 & 48 & 120 & $\mathrm{x}$ \\
\hline 9 & 7 & 18 & 25 & 31 & 39 & 97 & $\mathbf{x}$ \\
\hline 10 & 44 & 33 & 22 & 9 & 12 & 23 & $\mathrm{x}$ \\
\hline \multirow[t]{2}{*}{11} & 28 & 21 & 20 & 26 & 25 & 59 & \\
\hline & \multicolumn{5}{|c|}{ Set $E$} & & \\
\hline 1 & 0 & 12 & 24 & 36 & 48 & 120 & $\mathbf{x}$ \\
\hline 2 & 4 & 18 & 28 & 32 & 38 & 94 & $\mathrm{x}$ \\
\hline 3 & 18 & 32 & 32 & 22 & 16 & 52 & \\
\hline 4 & 0 & 17 & 26 & 36 & 41 & 110 & $\mathbf{x}$ \\
\hline 5 & 24 & 34 & 33 & 14 & 15 & 45 & $\mathbf{x}$ \\
\hline 6 & 21 & 18 & 22 & 33 & 26 & 73 & \\
\hline 7 & 10 & 19 & 27 & 29 & 35 & 87 & $\mathbf{x}$ \\
\hline 8 & 2 & 13 & 22 & 35 & 48 & 117 & $\mathrm{x}$ \\
\hline 9 & 9 & 17 & 22 & 36 & 36 & 99 & $\mathrm{x}$ \\
\hline 10 & 37 & 22 & 21 & 16 & 24 & 47 & \\
\hline \multirow[t]{2}{*}{11} & 2 & 19 & 25 & 31 & 43 & 103 & $\mathbf{X}$ \\
\hline & \multicolumn{5}{|c|}{ Set F } & & \\
\hline 1 & 0 & 13 & 25 & 34 & 47 & 118 & $\mathrm{x}$ \\
\hline 2 & 30 & 28 & 27 & 19 & 16 & 50 & \\
\hline 3 & 33 & 18 & 26 & 25 & 18 & 56 & \\
\hline 4 & 0 & 12 & 24 & 36 & 48 & 120 & $\mathbf{x}$ \\
\hline 5 & 17 & 13 & 26 & 29 & 35 & 87 & $\mathrm{x}$ \\
\hline 6 & 45 & 29 & 19 & 16 & 11 & 27 & $\mathrm{X}$ \\
\hline 7 & 16 & 33 & 30 & 25 & 16 & 55 & \\
\hline 8 & 0 & 12 & 24 & 37 & 47 & 119 & $\mathbf{x}$ \\
\hline 9 & 5 & 14 & 27 & 36 & 38 & 102 & $\mathrm{x}$ \\
\hline 10 & 47 & 31 & 21 & 15 & 6 & 14 & $\mathrm{x}$ \\
\hline 11 & 20 & 14 & 25 & 29 & 32 & 76 & $\mathrm{x}$ \\
\hline
\end{tabular}

The sum of the elements in this matrix was used to test the model. The sum equals 120 if a $\mathbf{S}$ chooses the thinner pair on every trial and it becomes 0 if a $S$ always chooses the more square rectangles. Under the hypothesis that $S$ has a preference probability of .5 for the more square stimuli, the sum should follow a binomial distribution with a mean of 60 .

Under this assumption, the sum of the matrix elements lies between 45 and 75 , with probability .99 . If it reaches or exceeds these boundaries, the hypothesis is rejected at the .01 level of significance.
To show the trend of a possible violation, it was determined for each pair how often it was chosen over any other pair. These values, together with the sums of the pair comparison matrices, are given in Table 2.

\section{DISCUSSION}

On the basis of the rating-scale data, the multidimensional scaling model was rejected for about half the Ss. Almost all Ss violated the model in the pair comparison experiment. The difference between the rating scale and the pair comparison data may be due to some methodological artifact. For example, the rating scale data may contain a larger amount of error.

As is shown in Tables 1 and 2, most of the Ss judged the overall difference to be larger for thin rectangles and to be smaller for squares. Furthermore, there was no systematic difference between the subexperiments. Thus, the hypothesis regarding context effects was not supported. For most Ss, the data appear very consistent. For some Ss, all 120 judgments of the pair comparison experiment are in the same direction. In a very few cases, judgments vary in an irregular manner, e.g., difference judgments being small for squares and thin rectangles and being larger for rectangles in between.

The main result of this study is that the predictions derived from decomposability and intradimensional subtractivity were violated by most of the Ss. Of course, it is possible that the violation of the model was caused by the special selection of stimuli and by the fact that not all possible pairs were presented throughout the experiment. Most probably Ss were aware of this and, accordingly, some response bias may have been introduced. Unfortunately, there is no way to control response bias in these types of scaling methods, and the multidimensional scaling model might hold for the same type of stimuli in different experimental conditions.

Though there remains the puzzling result that the multidimensional scaling model was violated by stimuli as simple as rectangles varying in area and shape, we cannot conclude that this will be the case when the model is applied to more complex stimuli. The stimuli used here are of the kind called analyzable by Shepard (1964). As suggested by Torgerson (1965), multidimensional scaling models might be more appropriate for unitary stimuli.

It is possible, at least in principle, that the model may be satisfied with the same stimuli, but with physical variables other than area and shape. Two obvious alternatives are height and width of rectangles. The unpublished study mentioned above, however, suggested that area and shape were the more relevant dimensions.

After this failure to confirm the model of multidimensional scaling, two alternatives remain. One is to completely reject the model and the other is to modify it. One modification that comes to mind is to redefine the psychophysical functions to include the interactions between the dimensions. But, from the point of view of economy, such a complication of the model seems to be undesirable.

\section{REFERENCES}

BEALS, R., \& KRANTZ, D. H. Metrics and geodesics induced by order relations. Mathematische Z eitschrift, 1967, 101 285-298.

BEALS, R., KRANTZ, D. H., \& TVERSKY, A Foundations of multidimensional scaling. Psychological Review, 1968, 75, 127-142.

PFANZAGL, J. Theory of measurement Würzburg: Physica, 1968.

SHEPARD, R. N. Attention and the metric structure of stimulus space. Journal of Mathematical Psychology, 1964, 1, 54-87.

STEVENS, S. S. Measurement, psychophysics and utility. In C. W. Churchman and P. Ratoosh (Eds.), Measurement definitions and theories. New York: Wiley, 1959. Pp. 18-63.

TORGERSON, W. S. Theory and method of scaling. New York: Wiley, 1958.

TORGERSON, W. S. Multidimensional scaling of similarity. Psychometrika, 1965, 30, 379-393.

TVERSKY, A., \& KRANTZ, D. H. Similarity of schematic faces: A test of interdimensional additivity. Perception \& Psychophysics, 1969, $5,124-128$.

(Accepted for publication November 16, 1970.) 